\title{
A STUDY OF STRESSORS, COPING SKILLS, AND BURDEN IN CAREGIVERS OF PATIENTS WITH SCHIZOPHRENIA
}

\author{
Rengarajulu Muralitharan ${ }^{1}$, Andisamy Niranjana Devi² \\ ${ }^{1}$ Associate Professor, Department of Psychiatry, MGMGH/KAPV Govt. Medical College, Tiruchirappalli. \\ ${ }^{2}$ Associate Professor, Department of Psychiatry, MGMGH/KAPV Govt. Medical College, Tiruchirappalli.
}

\section{ABSTRACT}

Schizophrenia is a severe mental illness and requires care for almost entire lifespan. Significant burden and stress are being experienced by the caregivers of patients with schizophrenia. Family members use various coping strategies in order to deal with the burden of caregiving. Assessing and reducing the caregivers' burden is important for the wellbeing of both caregivers and patients.

\section{AIM}

To assess coping strategies, levels of burden, and stressors experienced by caregivers of patients with schizophrenia and to identify the relationship between stressors, coping strategies, and burden among them.

\section{MATERIALS AND METHODS}

Convenience sampling method was used and the sample comprised of 30 caregivers of patients with schizophrenia. Presumptive Stressful Life Events Scale, Coping Check List, Burden Assessment Schedule were administered to study population. Data were analysed using appropriate statistical methods.

\section{RESULTS}

Among 30 study subjects, males and females participated in equal number with equal rural and urban participation. In relation to coping skills, males scored higher in problem solving and emotion focused coping strategies compared to females. The whole study group scored less in social support subscale of coping. $23.3 \%(n=7)$ of caregivers scored higher in total BAS score ( $>80)$ with wife as caregiver scored the highest. In relation to stressors, $23.3 \%(n=7)$ scored $>150$ life change units.

\section{CONCLUSION}

With mean duration of caregiving of $9.14 \pm 8.341$ years, the extent of burden in relatives of schizophrenic patients as caregiver was statistically significant among spouses, different age groups, and between rural and urban population. Among caregivers, mothers as subgroup ( $n=7)$ experienced lowest burden. Males, parents, and husbands as caregivers expressed higher coping skills. There was no statistically significant correlation found between stressful life events, coping strategy, and level of burden in this study.

\section{KEYWORDS}

Burden, Caregivers, Coping strategies, Schizophrenia.

HOW TO CITE THIS ARTICLE: Muralitharan R, Devi AN. A study of stressors, coping skills, and burden in caregivers of patients with schizophrenia. J. Evolution Med. Dent. Sci. 2016;5(55):3741-3749, DOI: 10.14260/jemds/2016/858

\section{INTRODUCTION}

Families are integral part of care system for persons with a chronic mental illness such as schizophrenia.1In India, more than $90 \%$ of the patients with chronic illness live with their families. $^{2}$ Weidmann et al. noted that despite a change in traditional family environment more than half of the patients with long-term schizophrenia live with at least one 'significant other' i.e. primary caregiver. ${ }^{3}$

Presence of individual with severe mental illness in the family causes stress, emotional disturbances, and strain on the family members, which is referred to as caregiver burden. ${ }^{4}$

Financial or Other, Competing Interest: None.

Submission 27-05-2016, Peer Review 29-06-2016,

Acceptance 04-07-2016, Published 09-07-2016.

Corresponding Author:

Dr. Rengarajulu Muralitharan,

Associate Professor,

Department of Psychiatry,

M. G. Memorial Government Hospital (MGMGH),

Tiruchirappalli-620017, Tamilnadu.

E-mail: drmuramind@gmail.com

DOI: $10.14260 /$ jemds $/ 2016 / 858$
Numerous studies have demonstrated that family caregivers of persons with severe mental illness suffer from significant stress, experience moderately high level of burden and often receive inadequate assistance from mental health professionals. $^{5}$

The demands of caring for a mentally ill relative have both an emotional and practical impact on the caregiver, ${ }^{6}$ which have been defined by concepts of subjective and objective burden. Change in the family role, activities, financial implications contributes to objective burden. Subjective burden includes the caregivers feeling of loss, guilt, and anger.7,8

Behavioural problem in patients adds on to the stress in caregivers. Thus, the caregiver experiences considerable stress and burden and need help in coping with it. ${ }^{9}$ Stress and strain caused by prolonged caregiving of mentally ill person is managed by various coping strategies. An unhealthy coping style is likely to adversely affect caregiving function.

Several variables in relation to patient and caregiver have been found to contribute to family burden. Not all caregivers perceive the same burden because it varies according to their ways of coping. Folkman and Lazarus have defined coping as 
a person's constantly changing cognitive and behavioural efforts to manage an encounter appraised as stressful. ${ }^{10}$

Stress can be defined as the response of the body to various physical and psychological demands, which may be internal or external and are perceived as threatening and out of their control and coping resources. ${ }^{11}$

Both emotion focused and problem focused coping lead to reappraisal of the stressful event, i.e. patients' illness. ${ }^{12}$ the emotion focused coping help to reduce emotional disturbances caused by the stressors by avoiding and refusing to accept the stressors and seeking religious help. The problem focused coping refers to direct actions, which the individual undertake to change the situation. These include problem solving or seeking social support to resolve the stress.13,14

Avoidance strategies seem to be less effective in regulating the distress of caregivers than problem-focused strategies. On the other hand, passive coping styles are associated with a high level of burden and problem solving coping styles are related to lower levels of burden in caregivers. $^{15}$ the caregivers' burden is determined significantly by their perception about the patient's condition than the illness per se. ${ }^{16}$

Those living in nuclear families are facing a new problem, because in the past, joint families provided human as well as material resources for the care of the people with mental illness. With increasing change in family structure from joint family to nuclear family with working parents have introduced newer problems of increasing load on the family caregivers. ${ }^{9}$

Caregivers' relationship with the mentally ill has a decisive effect on their burden. Many studies have documented various concerns expressed by parent caregiver (Not achieving educational, employment, marital goals). ${ }^{17}$ and spouse caregiver (Financial concerns, family relationships, and responsibilities such as child-rearing, planning for future). ${ }^{18}$

It has been noted by Pai and Kapur that caregiver's burden differed significantly between developed and under developed countries due to different sociocultural and economic backgrounds. The areas of burden and the pattern of accepting and rejecting patients in India may be entirely different.19 Wig et al. also found that expressed emotion as a concept associated with burden plays a relatively less significant role in Indian families. ${ }^{20}$

Among the stressors, patients' behavioural disturbances have stronger implications on caregiver burden. Patient's physical illness does not contribute much to the burden of caregivers of demented patients.

\section{MATERIAL AND METHOD}

The study was carried out on caregivers of patients diagnosed with schizophrenia, attending Department of Psychiatry, Mahatma Gandhi Memorial Government Hospital (MGMGH) attached to KAPV Govt. Medical College, Tiruchirappalli, were subjected for study.

With ethical clearance, this study involved assessment of subjects cross sectionally for once and a purposive sampling done.

Caregivers (Parents and spouses) who were above 18 years of age, actively involved in caregiving for at least 6 months prior to assessment were included in the study.
Caregivers with history of mental illness and who were cognitively impaired were excluded. This study included total of 30 caregivers of whom 14 were parents and 16 were spouses. Informed consent was obtained from all participants.

\section{Measures}

Socio-demographic data sheet was used to record name, age, sex, marital status, living status, area of living, and duration of caregiving.

Kuppusamy's socioeconomic status scale ${ }^{21}$ was applied to all subjects.

Burden Assessment Schedule (BAS, Thara et al., 1998). ${ }^{13}$ this is a 40 -item scale, which measures seven different areas of burden. Each item is rated on a three point scale with minimum score of 40 and maximum being 120 where the scoring corresponds to the burden level. The reliability value is 0.75 .

Coping checklist (Rao et al., 1989). ${ }^{14}$ this is a 70 item scale, in a Yes/No format. It covers a wide range of cognitive, behavioural, and emotional responses that are used to handle stress. There are seven subscales: problem solving, denial, positive distraction, negative distraction, acceptance, religion/faith, and social support seeking. The reliability value is 0.73 . Patients' coping resources are assessed based on the number of responses and checklist score.

Presumptive Stressful Life Events Scale (Gurmeet Singh et al. 1984).22 PSLES is a scale of life events designed for use in Indian population. The scale was revised based on Holmes and Rahe's Social Readjustment Rating Schedule. The scale consists of 51 items, which are arranged in ascending order of severity. Each event is given mean stress score that varies from 20 to 95 . The reliability value is 0.89 .

\section{Statistical Analyses}

Descriptive statistical data were analysed by Percentage, Mean and Standard Deviation. Results were analysed using Student t-test, one-way ANOVA and correlations were made with Karl-Pearson test.

\section{RESULTS}

In the total study population $(n=30)$, there were equal number of males $(n=15)$ and females $(n=15)$. The mean age of study population is $50.73 \pm 14.57$ with minimum age 28 and maximum age was 80 . Age group between 31-40 yrs. contributed $30 \%(n=9)$ and age group between $41-50$ yrs. constituted $26.7 \%(n=8)$.

In this study, spouses as caregivers were $53.4 \%(n=16)$, and parents were $46.6 \%(\mathrm{n}=14)$. Among the study group, $20 \%(n=6)$ were illiterate and $46.6 \%(n=14)$ attained primary and high school education. Regarding socio-economic status, 19 persons (63.3\%) were from lower/upper lower class and $9(30 \%)$ were from middle/lower middle class. In this study $73.3 \%(\mathrm{n}=22)$ were from nuclear family, remaining $26.7 \%$ $(n=8)$ from joint family, and equal number of caregivers were from rural and urban area. (Table-1).

The mean duration of caregiving is $9.74 \pm 8.34$ years $(1-30$ yrs.). Among caregivers $46.7 \%(n=14)$ were caregiving for less than 5 yrs. and $30 \%(n=9)$ were caring for 15 yrs. and above.

The mean number of stressful life events occurred in study population was $2.50 \pm .861$ (1-4 events). $50 \%(\mathrm{n}=15)$ of study population undergone 3 life events in the previous 
year. The mean life change units of overall study population is $110.13 \pm 47.03$ (0 to 202 life change units), $20 \%(n=6)$ had life change units between 150-200. Female caregivers $(n=15)$ had mean life change units of $120.20 \pm 46.07$ and male $(n=15)$ caregivers had $100.07 \pm 47.35$ life change units. Among females, mothers $(n=7)$ had mean life change units of $122.29 \pm$ 57.00. Though, the difference were statistically not significant. There was no statistically significant difference in life change units score among age groups and socio-economic status (Table- 2 and 3 ).

In this study, the mean total score for coping was $15.77 \pm 4.43$ (10 to 24 ), with higher mean score in problem solving coping $(4.37 \pm 1.06)$ and lower score for social support subtype of coping $(1.47 \pm 0.937)$. Males $(n=15)$ had mean score of $16.33 \pm 4.63$ and females $(n=15)$ with the score of $15.20 \pm 4.31$. In this study, males found to have higher mean score in the problem solving and emotion focused coping though the difference is statistically not significant. (Table-4).

Among different age group mean coping was highest in age group of less than 30 yrs. (21.00) and lowest in age group between 60 to 70 yrs. $(12.33 \pm 0.577)$ with respect to duration of caregiving, the study group with less than 2 yrs. of caregiving $(\mathrm{n}=6)$ was presented with high mean score (17.83 \pm 4.708$)$. (Table-5).

Compared to spouses $(15.38 \pm 4.129)$ parents had higher score of mean coping (16.21 \pm 4.886$)$. (Table-6).

In the assessment of burden in the caregiver, the total burden mean score was $66.57 \pm 12.878$ with the range of 41 to 92. $76.7 \%(n=23)$ scored between 41 to 80 and $23.3 \%(n=7)$ scored in the range of 81 to 120 . Males scored less mean burden score $62.40 \pm 11.070$ compared to females score of 70.73 \pm 13.557 . Among different age group, there was statistically significant difference in care givers burden.

Caregivers living in rural areas $(n=15)$ had higher burden score $(71.93 \pm 11.074)$ compared to caregivers from urban areas $(\mathrm{n}=15)$ who scored $(61.82 \pm 12.616)$ the difference being statistically significant. (Table-7, 8, 9).

Among the spouses, wives scored statistically significant difference burden score $(79.75 \pm 8.548)$ compared to husbands (62.75 \pm 12.23$)$. (Table-10).

In this study group, there was no statistically significant correlation between Stressors, Coping strategies, and Caregivers' Burden. (Table-11).

\begin{tabular}{|c|c|c|c|}
\hline \multirow{2}{*}{$\begin{array}{l}\text { Sl. } \\
\text { No. }\end{array}$} & & \multicolumn{2}{|c|}{ Total } \\
\hline & & $(n=30)$ & $100 \%$ \\
\hline \multirow[t]{5}{*}{1} & Below 30 & 1 & $3.3 \%$ \\
\hline & 31 to 40 yrs. & 9 & $30.0 \%$ \\
\hline & 41 to 50 yrs. & 8 & $26.7 \%$ \\
\hline & 51 to 70 yrs. & 7 & $23.3 \%$ \\
\hline & Above 70 yrs. & 5 & $16.7 \%$ \\
\hline \multirow[t]{3}{*}{2} & Sex & & \\
\hline & Male & 15 & $50 \%$ \\
\hline & Female & 15 & $50 \%$ \\
\hline \multirow[t]{7}{*}{3} & Education & & \\
\hline & Illiterate & 6 & $20.0 \%$ \\
\hline & Primary school & 7 & $23.3 \%$ \\
\hline & Middle school & 5 & $16.7 \%$ \\
\hline & High school & 7 & $23.3 \%$ \\
\hline & Diploma & 4 & $13.3 \%$ \\
\hline & UG & 1 & $3.3 \%$ \\
\hline \multirow[t]{6}{*}{4} & Occupation & & \\
\hline & Unskilled worker & 9 & $30.0 \%$ \\
\hline & Semi-skilled worker & 10 & $33.3 \%$ \\
\hline & Skilled worker & 5 & $16.7 \%$ \\
\hline & Clerical and Farmer & 5 & $16.7 \%$ \\
\hline & Semi-profession & 1 & $3.3 \%$ \\
\hline
\end{tabular}

\begin{tabular}{|c|c|c|c|}
\hline \multirow{2}{*}{$\begin{array}{c}\text { Sl. } \\
\text { No. }\end{array}$} & & \multicolumn{2}{|c|}{ Total } \\
\hline & & $(n=30)$ & $100 \%$ \\
\hline \multirow[t]{5}{*}{5} & Income & & \\
\hline & Rs. 1601 to 4809 & 15 & $50 \%$ \\
\hline & Rs. 4810 to 8009 & 6 & $20.0 \%$ \\
\hline & Rs. 8010 to 12019 & 5 & 16.7 \\
\hline & Rs. 12020 to 32049 & 4 & $13.3 \%$ \\
\hline \multirow[t]{4}{*}{6} & Socio-Economic Status & & \\
\hline & Upper middle & 2 & $6.7 \%$ \\
\hline & Middle \lower middle & 9 & $30.0 \%$ \\
\hline & Lower \upper lower & 19 & $63.3 \%$ \\
\hline \multirow[t]{3}{*}{7} & Relationship of the Caregivers & & \\
\hline & Parent & 14 & $46.6 \%$ \\
\hline & Spouse & 16 & $53.4 \%$ \\
\hline \multirow[t]{3}{*}{8} & Living Status & & \\
\hline & Nuclear family & 8 & $26.7 \%$ \\
\hline & Joint family & 22 & $73.3 \%$ \\
\hline \multirow[t]{3}{*}{9} & Area of Living & & \\
\hline & Rural & 15 & $50 \%$ \\
\hline & Urban & 15 & $50 \%$ \\
\hline \multirow[t]{4}{*}{10} & Religion & & \\
\hline & Hindu & 23 & $76.7 \%$ \\
\hline & Muslim & 3 & $10 \%$ \\
\hline & Christian & 4 & $13.3 \%$ \\
\hline
\end{tabular}

\begin{tabular}{|c|c|c|c|c|c|}
\hline Sl. No. & Item & Min. & Max. & Mean & S.D. \\
\hline 1 & Age & 28 & 80 & 50.73 & 14.572 \\
\hline 2 & Duration of Care & 1 & 30 & 9.74 & 8.341 \\
\hline 3 & PSLES - Number of events & 0 & 4 & 2.50 & .861 \\
\hline 4 & PSLES- Life event score & 0 & 202 & 110.13 & 47.034 \\
\hline 5 & CCL (Coping Check List) - Total & 2 & 24 & 15.77 & 4.439 \\
\hline 6 & CCL - problem solving & 6 & 4.37 & 1.066 \\
\hline
\end{tabular}




\begin{tabular}{|c|c|c|c|c|c|}
\hline 7 & CCL - Positive distraction & 0 & 6 & 2.33 & 1.398 \\
\hline 8 & CCL - Negative Distraction & 1 & 4 & 2.33 & 0.922 \\
\hline 9 & CCL - Acceptance & 0 & 4 & 1.90 & 0.995 \\
\hline 10 & CCL - Religion & 0 & 4 & 1.50 & 1.106 \\
\hline 11 & CCL - Denial & 0 & 4 & 1.80 & 1.031 \\
\hline 12 & CCL - Social support & 0 & 4 & 1.47 & 0.937 \\
\hline 13 & BAS (Burden Assessment Scale) - Total & 0 & 12 & 5.17 & 5.100 \\
\hline 14 & BAS - Spouse related & 6 & 15 & 10.70 & 1.915 \\
\hline 15 & BAS - Physical and Mental Health & 5 & 12 & 8.97 & 2.042 \\
\hline 16 & BAS - External support & 7 & 12 & 9.13 & 1.634 \\
\hline 17 & BAS - Caregiver routine & 4 & 11 & 7.30 & 1.950 \\
\hline 18 & BAS - support of patient & 3 & 8 & 5.33 & 1.539 \\
\hline 19 & BAS - Taking responsibilities & 5 & 10 & 7.60 & 1.610 \\
\hline 20 & BAS - Other relations & 4 & 10 & 5.80 & 1.518 \\
\hline 21 & BAS - Patient behaviour & & & \\
\hline 22 & BAS - Caregiver Strategy & & \\
\hline
\end{tabular}

\begin{tabular}{|c|c|c|c|}
\hline PSLES - Life Event Score & Mean & S.D & Statistical Inference \\
\hline Male $(n=15)$ & 100.07 & 47.353 & \multirow{2}{*}{$\begin{array}{c}\mathrm{T}=-1.180 \mathrm{Df}=28 \\
.248>0.05^{*}\end{array}$} \\
\hline Female $(n=15)$ & 120.20 & 46.075 & \\
\hline Father $(n=7)$ & 92.57 & 54.218 & \multirow{2}{*}{$\begin{array}{c}\mathrm{T}=-.999 \mathrm{Df}=12 \\
.337>0.05^{*}\end{array}$} \\
\hline Mother $(n=7)$ & 122.29 & 57.002 & \\
\hline Husband $(\mathrm{n}=8)$ & 106.63 & 43.124 & \multirow{2}{*}{$\begin{array}{c}\mathrm{T}=-.577 \mathrm{Df}=14 \\
.573>0.05^{*}\end{array}$} \\
\hline Wife $(n=8)$ & 118.38 & 38.112 & \\
\hline Parents (n=14) & 107.43 & 55.625 & \multirow{2}{*}{$\begin{array}{c}\mathrm{T}=-.290 \mathrm{Df}=28 \\
.774>0.05^{*}\end{array}$} \\
\hline Spouse $(n=16)$ & 112.50 & 39.781 & \\
\hline
\end{tabular}

\begin{tabular}{|c|c|c|c|c|c|c|}
\hline PSLES - Life Event Score & Mean & S.D. & SS & DF & MS & Statistical Inference \\
\hline Between age Groups & & & 9460.903 & 5 & 1892.181 & \multirow{8}{*}{$\begin{array}{c}\mathrm{F}=.830 \\
.541>0.05^{*}\end{array}$} \\
\hline Below $30(n=1)$ & 108.00 & . & & & & \\
\hline 31 to $40(n=9)$ & 118.11 & 43.676 & & & & \\
\hline 41 to $50(n=8)$ & 126.88 & 44.418 & & & & \\
\hline 51 to $60(n=4)$ & 115.00 & 65.258 & & & & \\
\hline 60 to $70(n=3)$ & 74.00 & 9.849 & & & & \\
\hline Above $70(n=5)$ & 87.20 & 56.238 & & & & \\
\hline Within Groups & & & 54692.564 & 24 & 2278.857 & \\
\hline Between Socio-Economic Groups & & & 11084.230 & 2 & 5542.115 & \multirow{5}{*}{$\begin{array}{c}\mathrm{F}=2.820 \\
.077>0.05^{*}\end{array}$} \\
\hline Upper middle $(\mathrm{n}=2)$ & 38.50 & 54.447 & & & & \\
\hline Middle $\backslash$ lower middle $(\mathrm{n}=9$ ) & 112.67 & 37.759 & & & & \\
\hline Lower\upper lower $(\mathrm{n}=19)$ & 116.47 & 46.367 & & & & \\
\hline Within Groups & & & 53069.237 & 27 & 1965.527 & \\
\hline \multicolumn{7}{|c|}{ Table 3: Life Events Score Among Different Groups } \\
\hline
\end{tabular}

*Not significant

\begin{tabular}{|c|c|c|c|}
\hline Variables & $\begin{array}{c}\text { Male (n=15) } \\
\text { Mean } \pm \text { S.D }\end{array}$ & $\begin{array}{c}\text { Female (n=15) } \\
\text { Mean } \pm \text { S.D }\end{array}$ & Significance \\
\hline Problem solving & $4.47 \pm 1.125$ & $4.27 \pm 1.033$ & $\begin{array}{c}\mathrm{T}=.507 \mathrm{Df}=28 \\
.616>0.05^{*}\end{array}$ \\
\hline Positive distraction & $2.67 \pm 1.676$ & $2.00 \pm 1.000$ & $\begin{array}{c}\mathrm{T}=1.323 \mathrm{Df}=28 \\
.197>0.05^{*}\end{array}$ \\
\hline Negative distraction & $2.27 \pm 961$ & $2.40 \pm 910$ & $\begin{array}{c}\mathrm{T}=-.390 \mathrm{Df}=28 \\
.699>0.05^{*}\end{array}$ \\
\hline Acceptance & $2.07 \pm 961$ & $1.73 \pm 1.033$ & $\begin{array}{c}\mathrm{T}=.915 \mathrm{Df}=28 \\
.368>0.05^{*}\end{array}$ \\
\hline
\end{tabular}




\begin{tabular}{|c|c|c|c|}
\hline Religion & $1.53 \pm 1.125$ & $1.47 \pm 1.125$ & $\begin{array}{c}\mathrm{T}=.162 \mathrm{Df}=28 \\
.872>0.05^{*}\end{array}$ \\
\hline Denial & $1.93 \pm 1.033$ & $1.67 \pm 1.047$ & $\begin{array}{c}\mathrm{T}=.702 \mathrm{Df}=28 \\
.488>0.05^{*}\end{array}$ \\
\hline Emotion focused & $10.47 \pm 3.583$ & $9.40 \pm 3.397$ & $\begin{array}{c}\mathrm{T}=.837 \mathrm{Df}=28 \\
.410>0.05^{*}\end{array}$ \\
\hline Social support & $1.40 \pm 986$ & $1.53 \pm 915$ & $\begin{array}{c}\mathrm{T}=-.384 \mathrm{Df}=28 \\
.704>0.05^{*}\end{array}$ \\
\hline \multicolumn{2}{r}{} \\
\hline
\end{tabular}

*Not significant

\begin{tabular}{|c|c|c|c|c|c|c|c|}
\hline \multirow{2}{*}{ Variable } & \multirow{2}{*}{ CCL-Total } & \multicolumn{2}{|c|}{ CCL_TOTAL } & \multirow{2}{*}{ SS } & \multirow{2}{*}{ DF } & \multirow{2}{*}{ MS } & \multirow{2}{*}{$\begin{array}{l}\text { Statistical } \\
\text { Inference }\end{array}$} \\
\hline & & Mean & S.D & & & & \\
\hline \multirow{8}{*}{ Age } & Between Groups & & & 70.625 & 5 & 14.125 & \multirow{8}{*}{$\begin{array}{c}\mathrm{F}=.677 \\
.645>0.05^{*}\end{array}$} \\
\hline & Below $30(n=1)$ & 21.00 & & & & & \\
\hline & 31 to $40(n=9)$ & 15.67 & 4.796 & & & & \\
\hline & 41 to $50(n=8)$ & 16.13 & 4.224 & & & & \\
\hline & 51 to $60(n=4)$ & 17.00 & 5.831 & & & & \\
\hline & 60 to $70(n=3)$ & 12.33 & 0.577 & & & & \\
\hline & Above $70(n=5)$ & 15.40 & 4.722 & & & & \\
\hline & Within Groups & & & 500.742 & 24 & 20.864 & \\
\hline \multirow{7}{*}{$\begin{array}{l}\text { Duration } \\
\text { of care }\end{array}$} & Between Groups & & & 46.858 & 4 & 11.715 & \multirow{7}{*}{$\begin{array}{c}\mathrm{F}=.558 \\
.695>0.05^{*}\end{array}$} \\
\hline & Below 2 yrs. $(n=6)$ & 17.83 & 4.708 & & & & \\
\hline & 2 to 5 yrs. $(n=8)$ & 15.13 & 4.673 & & & & \\
\hline & 5 to 10 yrs. $(n=5)$ & 16.20 & 5.167 & & & & \\
\hline & 10 to 15 yrs. $(n=2)$ & 13.00 & .000 & & & & \\
\hline & 15 yrs. and above $(n=9)$ & 15.33 & 4.387 & & & & \\
\hline & Within Groups & & & 524.508 & 25 & 20.980 & \\
\hline & & & & & & & \\
\hline \multirow{5}{*}{$\begin{array}{c}\text { Socio Economic } \\
\text { Class }\end{array}$} & Between Groups & & & 14.680 & 2 & 7.340 & \multirow{5}{*}{$\begin{array}{c}\mathrm{F}=.356 \\
.704>0.05^{*}\end{array}$} \\
\hline & Upper middle $(\mathrm{n}=2)$ & 14.50 & 3.536 & & & & \\
\hline & Middle \lower middle $(\mathrm{n}=9)$ & 16.78 & 4.944 & & & & \\
\hline & Lower\upper lower $(n=19)$ & 15.42 & 4.401 & & & & \\
\hline & Within Groups & & & 556.687 & 27 & 20.618 & \\
\hline & Table 5: Co & ison of & ping & ng Stud & oups & & \\
\hline
\end{tabular}

*Not significant

\begin{tabular}{|c|c|c|c|}
\hline CCL-Total & Mean & S.D & Statistical Inference \\
\hline Male $(n=15)$ & 16.33 & 4.639 & \multirow{2}{*}{$\begin{array}{c}\mathrm{T}=.693 \mathrm{Df}=28 \\
.494>0.05^{*}\end{array}$} \\
\hline Female $(n=15)$ & 15.20 & 4.313 & \\
\hline Father $(n=7)$ & 16.71 & 4.957 & \multirow{2}{*}{$\begin{array}{c}\mathrm{T}=.370 \mathrm{Df}=12 \\
.718>0.05^{*}\end{array}$} \\
\hline Mother $(n=7)$ & 15.71 & 5.155 & \\
\hline Husband $(\mathrm{n}=8)$ & 16.00 & 4.660 & \multirow{2}{*}{$\begin{array}{c}\mathrm{T}=.592 \mathrm{Df}=14 \\
.563>0.05^{*}\end{array}$} \\
\hline Wife $(n=8)$ & 14.75 & 3.732 & \\
\hline Parents $(n=14)$ & 16.21 & 4.886 & \multirow{2}{*}{$\begin{array}{c}\mathrm{T}=.510 \mathrm{Df}=28 \\
.614>0.05^{*}\end{array}$} \\
\hline Spouse $(n=16)$ & 15.38 & 4.129 & \\
\hline Rural (n=15) & 16.00 & 4.943 & \multirow{2}{*}{$\begin{array}{c}\mathrm{T}=.283 \mathrm{Df}=28 \\
.779>0.05^{*}\end{array}$} \\
\hline Urban $(n=15)$ & 15.53 & 4.033 & \\
\hline Joint family (n=8) & 17.13 & 4.734 & \multirow{2}{*}{$\begin{array}{c}\mathrm{T}=1.011 \mathrm{Df}=28 \\
>0.05^{*}\end{array}$} \\
\hline Nuclear family $(n=22)$ & 15.27 & 4.333 & \\
\hline
\end{tabular}

*Not significant

\begin{tabular}{|c|c|c|c|}
\hline \multirow{2}{*}{ Variable } & \multicolumn{2}{|c|}{ BAS - Total } & \multirow{2}{*}{ Statistical Inference } \\
\cline { 2 - 4 } & Mean & S.D. & T $=-1.844 \mathrm{Df}=28$ \\
\hline Male $(\mathrm{n}=15)$ & 62.40 & 11.070 & $.076>0.05^{*}$ \\
\hline Female $(\mathrm{n}=15)$ & 70.73 & 13.557 & \\
\hline
\end{tabular}




\begin{tabular}{|c|c|c|c|}
\hline Father $(n=7)$ & 62.00 & 10.536 & \multirow{2}{*}{$\begin{array}{c}\mathrm{T}=.279 \mathrm{Df}=12 \\
.785>0.05^{*}\end{array}$} \\
\hline Mother $(n=7)$ & 60.43 & 10.549 & \\
\hline Husband $(\mathrm{n}=8)$ & 62.75 & 12.233 & \multirow{2}{*}{$\begin{array}{c}\mathrm{T}=-3.222 \mathrm{Df}=14 \\
.006<0.05 \text { Significant }\end{array}$} \\
\hline Wife $(n=8)$ & 79.75 & 8.548 & \\
\hline Parents $(n=14)$ & 61.21 & 10.162 & \multirow{2}{*}{$\begin{array}{c}\mathrm{T}=-.101 \mathrm{Df}=28 \\
.920>0.05^{*}\end{array}$} \\
\hline Spouse $(n=16)$ & 61.63 & 11.860 & \\
\hline Rural (n=15) & 71.93 & 11.074 & \multirow{2}{*}{$\begin{array}{c}\mathrm{T}=2.476 \mathrm{Df}=28 \\
.020<0.05 \text { Significant }\end{array}$} \\
\hline $\operatorname{Urban}(n=15)$ & 61.20 & 12.616 & \\
\hline Joint family $(n=8)$ & 63.13 & 11.655 & \multirow{2}{*}{$\begin{array}{c}\mathrm{T}=-.879 \mathrm{Df}=28 \\
.387>0.05^{*}\end{array}$} \\
\hline Nuclear family $(n=22)$ & 67.82 & 13.326 & \\
\hline
\end{tabular}

${ }^{*}$ Not significant

\begin{tabular}{|c|c|c|c|c|c|c|c|}
\hline \multirow{2}{*}{ Variable } & & \multicolumn{2}{|c|}{ BAS - Total } & \multirow{2}{*}{ SS } & \multirow{2}{*}{ DF } & \multirow{2}{*}{ MS } & \multirow{2}{*}{$\begin{array}{l}\text { Statistical } \\
\text { Inference }\end{array}$} \\
\hline & & Mean & S.D. & & & & \\
\hline \multirow{8}{*}{ Age } & Between Groups & & & 1868.625 & 5 & 373.725 & \multirow{8}{*}{$\begin{array}{c}\mathrm{F}=3.050 \\
.029<0.05 \\
\text { Significant }\end{array}$} \\
\hline & Below $30(n=1)$ & 81.00 & & & & & \\
\hline & 31 to $40(n=9)$ & 75.33 & 13.509 & & & & \\
\hline & 41 to $50(n=8)$ & 59.88 & 10.535 & & & & \\
\hline & 51 to $60(n=4)$ & 71.00 & 4.082 & & & & \\
\hline & 60 to $70(n=3)$ & 64.33 & 9.452 & & & & \\
\hline & Above $70(n=5)$ & 56.40 & 10.900 & & & & \\
\hline & Within Groups & & & 2940.742 & 24 & 122.531 & \\
\hline \multirow{5}{*}{$\begin{array}{c}\text { Socio-Economic } \\
\text { Level }\end{array}$} & Between Groups & & & 224.864 & 2 & 112.432 & \multirow{5}{*}{$\begin{array}{c}\mathrm{F}=.662 \\
.524>0.05^{*}\end{array}$} \\
\hline & Upper middle $(\mathrm{n}=2)$ & 57.00 & 0.000 & & & & \\
\hline & Middle $\backslash$ lower middle $(\mathrm{n}=9)$ & 65.78 & 12.081 & & & & \\
\hline & Lower\upper lower $(\mathrm{n}=19)$ & 67.95 & 13.778 & & & & \\
\hline & Within Groups & & & 4584.503 & 27 & 169.796 & \\
\hline \multirow{7}{*}{$\begin{array}{c}\text { Duration } \\
\text { of } \\
\text { Care }\end{array}$} & Between Groups & & & 1074.403 & 4 & 268.601 & \multirow{7}{*}{$\begin{array}{c}\mathrm{F}=1.798 \\
.161>0.05^{*}\end{array}$} \\
\hline & Below 2 yrs. $(n=6)$ & 61.00 & 14.683 & & & & \\
\hline & 2 to 5 yrs. $(n=8)$ & 72.88 & 14.808 & & & & \\
\hline & 5 to 10 yrs. $(n=5)$ & 70.60 & 7.162 & & & & \\
\hline & 10 to 15 yrs. $(n=2)$ & 51.00 & 14.142 & & & & \\
\hline & 15 yrs. and above $(n=9)$ & 65.89 & 9.466 & & & & \\
\hline & Within Groups & & & 3734.964 & 25 & 149.399 & \\
\hline
\end{tabular}

*Not significant

\begin{tabular}{|l|c|c|c|}
\hline \multicolumn{1}{|c|}{ Variables } & $\begin{array}{c}\text { MALE (n=15) } \\
\text { Mean } \pm \text { SD }\end{array}$ & $\begin{array}{c}\text { FEMALE (n=15) } \\
\text { Mean } \pm \text { SD }\end{array}$ & Significance \\
\hline 1. Total BAS & $62.40 \pm 11.070$ & $70.73 \pm 13.557$ & $\begin{array}{c}\mathrm{t}=-1.844 \mathrm{df}=28 \\
.076>0.05^{*}\end{array}$ \\
\hline 2. Mental and Physical health & $10.07 \pm 1.438$ & $11.33 \pm 2.160$ & $\begin{array}{c}\mathrm{t}=-1.891 \mathrm{df}=28 \\
.069>0.05^{*}\end{array}$ \\
\hline 3. External support & $8.27 \pm 2.282$ & $9.67 \pm 1.543$ & $\begin{array}{c}\mathrm{t}=-1.968 \mathrm{df}=28 \\
.059>0.05^{*}\end{array}$ \\
\hline 4. Caregiver routine & $8.87 \pm 1.846$ & $9.40 \pm 1.404$ & $\begin{array}{c}\mathrm{t}=-.890 \mathrm{df}=28 \\
.381>0.05^{*}\end{array}$ \\
\hline 5. Support of patient & $6.07 \pm 1.534$ & $7.13 \pm 1.552$ & $\begin{array}{c}\mathrm{t}=-1.893 \mathrm{df}=28 \\
.069>0.05^{*}\end{array}$ \\
\hline 6. Taking responsibilities & $6.53 \pm 1.685$ & $8.07 \pm 1.944$ & $\begin{array}{c}\mathrm{t}=-2.308 \mathrm{df}=28 \\
.029<0.05 \\
\text { Significant }\end{array}$ \\
\hline 7. Other relations & & $\begin{array}{c}\mathrm{t}=-.706 \mathrm{df}=28 \\
.486>0.05^{*}\end{array}$ \\
\hline
\end{tabular}




\begin{tabular}{|l|c|c|c|}
\hline 8. Patient behaviour & $7.40 \pm 1.549$ & $7.80 \pm 1.699$ & $\begin{array}{c}\mathrm{t}=-.674 \mathrm{df}=28 \\
.506>0.05^{*}\end{array}$ \\
\hline 9. Care giver strategies & $5.47 \pm 1.598$ & $6.13 \pm 1.407$ & $\begin{array}{c}\mathrm{t}=-1.213 \mathrm{df}=28 \\
.235>0.05^{*}\end{array}$ \\
\hline \multicolumn{2}{|c|}{ Table 9: Comparison of Burden Among MALE and FEMALE } \\
\hline
\end{tabular}

*Not significant

\begin{tabular}{|c|c|c|c|}
\hline \multirow{2}{*}{ Variables } & Husband $(n=8)$ & Wife $(n=8)$ & \multirow{2}{*}{ Significance } \\
\hline & Mean \pm S.D & Mean \pm S.D. & \\
\hline 1. Total BAS & $62.75 \pm 12.233$ & $79.75 \pm 8.548$ & $\begin{array}{c}\mathrm{T}=-3.222 \mathrm{Df}=14 \\
.006<0.05 \\
\text { Significant }\end{array}$ \\
\hline 2. Spouse related & $8.63 \pm 1.768$ & $10.75 \pm 1.389$ & $\begin{array}{c}\mathrm{T}=-2.674 \mathrm{Df}=14 \\
.018<0.05 \\
\text { Significant }\end{array}$ \\
\hline 3. Mental and Physical health & $9.50 \pm 1.414$ & $12.13 \pm 1.885$ & $\begin{array}{c}\mathrm{T}=-3.151 \mathrm{Df}=14 \\
.007<0.05 \\
\text { Significant }\end{array}$ \\
\hline 4. External support & $8.25 \pm 2.493$ & $10.38 \pm 916$ & $\begin{array}{c}\mathrm{T}=-2.263 \mathrm{Df}=14 \\
.040<0.05 \\
\text { Significant }\end{array}$ \\
\hline 5. Caregiver routine & $8.38 \pm 1.506$ & $10.00 \pm 1.195$ & $\begin{array}{c}\mathrm{T}=-2.391 \mathrm{Df}=14 \\
.031<0.05 \\
\text { Significant }\end{array}$ \\
\hline 6. Support of patient & $5.88 \pm 1.553$ & $7.00 \pm 1.414$ & $\begin{array}{c}\mathrm{T}=-1.515 \mathrm{Df}=14 \\
.152>0.05 \\
\text { Not Significant }\end{array}$ \\
\hline 7. Taking responsibilities & $5.88 \pm 1.458$ & $8.75 \pm 1.488$ & $\begin{array}{c}\mathrm{T}=-3.904 \mathrm{Df}=14 \\
.002<0.05 \\
\text { Significant }\end{array}$ \\
\hline 8. Other relations & $4.50 \pm 2.000$ & $6.25 \pm 886$ & $\begin{array}{c}\mathrm{T}=-2.263 \mathrm{Df}=14 \\
.040<0.05 \\
\text { Significant }\end{array}$ \\
\hline 9. Patient behaviour & $6.88 \pm 1.246$ & $8.63 \pm 916$ & $\begin{array}{c}\mathrm{T}=-3.200 \mathrm{Df}=14 \\
.006<0.05 \\
\text { Significant }\end{array}$ \\
\hline 10. Caregiver strategies & $4.88 \pm 991$ & $6.00 \pm 1.512$ & $\begin{array}{c}\mathrm{T}=-1.760 \mathrm{Df}=14 \\
.100>0.05 \\
\text { Not Significant }\end{array}$ \\
\hline & Comparison c & Spouse & \\
\hline
\end{tabular}

\begin{tabular}{|c|c|c|}
\hline Variables & Correlation (r value) & P value \\
\hline BAS vs CCL & -.054 & .776 (Not Significant) \\
\hline BAS vs Stressors (Life Event Score) & .456 & .133 (Not Significant) \\
\hline CCL vs Stressors (Life Event Score) & .217 & .249 (Not Significant) \\
\hline \multicolumn{2}{|c|}{ Table 11: Correlation Between Burden, Coping Strategies, and Stressors } \\
\hline
\end{tabular}

\section{DISCUSSION}

The study examined the difference in the experience of stressor, burden, and coping styles in the caregivers, and the role of demographic variables, stressors, and coping strategies of caregivers in contributing to burden.

The Socio-Demographic data of the caregivers indicate $60 \%(n=18)$ of the study group were less than 50 yrs. of age and $40 \%(n=12)$ were above 50 yrs. In this study, males and females are equally represented. This finding was in contrast to majority of the studies on caregivers where females were largely represented.15,23,24 The mean age of the study group was $50.73 \pm 14.572$ and duration of care was $9.74 \pm 8.341$ where as in the study of Wageeh Abdel-Nasser Hassan et al 15 the mean age was $33.6 \pm 11.6$ and in the study of Anupama Rammohan et al25 the mean age of parents and spouses was $54.4 \pm 7.96$ and $47.29 \pm 8.07$, the mean duration of the care in that study was $5.88 \pm 4.59$ and $7.50 \pm 4.97$ for parents and 
spouses as caregivers, in the study of Vasudeva $\mathrm{S}$ et al. ${ }^{25}$ the mean age of caregiver was $48.32 \pm 11.65$ and duration of caregiving by parents was $7.13 \pm 3.13$.

In this study, equal number $(\mathrm{n}=15)$ of caregivers from rural and urban areas were participated. This was contrary to the study done by Dean A. Creado et $\mathrm{al}^{23}$ and study of Vasudeva $S$ et al.25 where more from urban setting participated. $73.3 \%(\mathrm{n}=22)$. Caregivers from nuclear family setup participated more, which is in agreement with study done by Vasudeva $\mathrm{S}$ et $\mathrm{al}^{25}$ and Dean $\mathrm{A}$. Creado et $\mathrm{al}^{23}$

The study shows that families of schizophrenia experience considerable burden. The mean burden score $66.57 \pm 12.878$ was in agreement with study done by Vasudeva $S$ et $\mathrm{al}^{25}(68.75 \pm 10.16)$ and study of Anupama Rammohan et al24 $(65.50 \pm 8.61$ for parents and $70.00 \pm 8.74$ for spouses), and not in agreement with Dean A. Creado et al 23 (78.52 \pm 12.70 in group A and $68.13 \pm 11.37$ in group B). In our study, $23.3 \%(n=7)$ scored higher burden i.e. $>80$ in BAS total, which is not consistent with the study by Dean A. Creado et $\mathrm{al}^{23}$ where $60 \%$ in group $\mathrm{A}$ and $28 \%$ in group $\mathrm{B}$ experienced higher burden.

Among study group, parents scored burden mean score of $61.21 \pm 10.16$ and spouses scored $61.63 \pm 11.86$. This finding is lower than observation made in the study of Anupama Rammohan et $\mathrm{al}^{24}(65.50 \pm 8.61$ in parents and 70.00 \pm 8.74 in spouses).

For spouse caregivers, physical impairments and patients' behaviour problems had a stronger relationship to burden. ${ }^{26}$ In this study, wives as caregivers scored highest burden (79.75 \pm 8.548$)$ and mothers scored lowest burden (60.43 \pm 10.549). When compared to males, females scored higher mean burden in all areas. Within spouses, wives scored statistically significant higher burden except in areas of caregivers' strategy and support of the patient.

The literature on stressful life events devotes far less attention to continuous or chronic stressors than to discrete events (Kessler, Price, and Wortman, 1985). ${ }^{27}$ Yet, in surveying the most widely used scales, Angermeyer (1985). ${ }^{28}$ pointed out, "You will find every major event conceivable in an individual's life, but you will miss the fact that a close relative has become mentally ill, one of the most devastating and catastrophic events that they can experience."

\section{Limitations}

Some limitations of this study are sample size and crosssectional design. A longitudinal prospective study would give more information about the factors studied.

\section{Implications}

In developing countries like ours, mentally ill patients are primarily taken care in family setup. Therefore, this study aids in understanding caregivers' burden, various strategies of coping used by them, and its impact over their burden.

\section{Future Directions}

In the era of deinstitutionalisation in spite of social significance, research on caregivers' burden is meager. ${ }^{29}$ with more number of studies, one can understand the burden and disruption caused by the mentally ill on the caregivers. The caregivers often benefit from counseling that provide emotional support and education that improves their understanding about the patient illness and its management.
By this, we can improve their problem focused coping and there by their positive well-being.

\section{CONCLUSION}

Mental health professionals should give due concern to caregiver burden and associated distress while evaluating and treating chronic mentally ill persons. Early identification and suitable interventions would help in keeping this support base intact, healthy, and effective. This would help the caregivers to deal effectively with the burden of caregiving using healthy coping strategies and also improve their caregiving capacity. ${ }^{9}$

\section{REFERENCES}

1. Shankar R, Menon S. Development of framework of interventions with families of people in the management of schizophrenia. Psychosocial Rehabilitation Journal 1993;16(3):75-91.

2. Chadda RK. Psychiatric patient in the community: challenges and solutions. J Ment Health Behave 2001;6:7-15.

3. Natalie $\mathrm{C}$, Ian $\mathrm{MD}$, Steve $\mathrm{H}$, et al. Measuring chronic patients feelings of being a burden to caregivers: development and preliminary validation of a schizophrenia. PLoS Med 2005;2:e141.

4. Saunders JC. Families living with severe mental illness: a literature review. Issues Ment Health Nurs 2003;24(2):175-98.

5. Weidmann G, Halweg K, Hank G, et al. Deliverability of psycho-educational social family management. Schizophr Bull 1994;20(3):547-56.

6. Chakrabarti S, Raj L, Kulhara F, et al. Comparison of the extent and patterns of family burden in affective disorders and schizophrenia. Indian J Psychiatry 1995;37(3):105-12.

7. Hoenig J, Hamilton MW. The schizophrenic patient in the community and his effect on the house hold. Int J Soc Psychiatry 1966;12(3):165-76.

8. Platt S. Measuring the burden of psychiatric illness on the family: an evaluation of some rating scales. Psychol Med 1985;15(2):383-93.

9. Chadda RK. Caring for the family caregivers of persons with mental illness. Indian J Psychiatry 2014;56(3): 221-7.

10. Folkman S, Lazarus RS. If it changes it must be a process: study of emotion and coping during three stages of a college examination. J Personality and Social Psychology 1985;48(1):150-70.

11. Morgan CT, King RA, Weisz, et al. Introduction to Psychology. $7^{\text {th }}$ edn. Newdelhi: Tata McGraw-Hill 1993.

12. Lazarus RS, Folkman S. Stress, appraisal, and coping. New York: Springer 1984.

13. Thara R, Padmavati R, Kumar S, et al. Burden assessment schedule: instrument to assess burden on caregivers of chronic mentally ill. Indian J Psychiatry 1998;40(1):21-9.

14. Rao K, Subbakrishna DK, Prabu GG. Development of a coping checklist: a preliminary report. Indian J Psychiatry 1989;31(2):128-33.

15. Hassan NWA, Mohamed II, Elnaser AEA, et al. Burden and coping strategies in caregivers of schizophrenic patients. Journal of American Science 2011;7(5):802-11. 
16. Scazufca M, Kuipers E. Links between expressed emotion and burden of care in relatives of patients with schizophrenia. Br J Psychiatry 1996;168(5):580-7.

17. Pickett SA, Cook JA, Cohler BJ. Caregiving burden experienced by parents of offspring with severe mental illness: the impact off-timedness. Journal of Applied Social Sciences 1994;18:199-207.

18. Mannion E. Resilience and burden in spouses of people with mental illness. Psychiatric Rehabilitation Journal 1996;20(2):13-23.

19. Pai $S$, Kapur RL. Burden on the family of a psychiatric patient: development of an interview schedule. $\mathrm{Br} \mathrm{J}$ Psychiatry 1981;138(4):332-5.

20. Wig NN, Menon DK, Bedi H, et al. Expressed emotion and schizophrenia in North India. I. cross-cultural transfer of ratings of relatives expressed emotion. Br J Psychiatry 1987;151(2):156-60.

21. Kumar RBP, Dudala SR, Rao AR. Kuppusamy's socioeconomic status scale: a revision of economic parameter for 2012. International Journal of Research and Development of Health 2013;1(1):2-4.

22. Singh G, Kaur D, Kaur H. Presumptive stressful life events scale: a new stressful life events scale for use in India. Indian J of Psychiatry 1984;26(2):107-14.

23. Credo DA, Parker SR, Kamath RM, et al. A comparison of the level of function in chronic schizophrenia with coping and burden in caregivers. Indian J of Psychiatry 2006;48(1):27-33.
24. Rammohan A, Rao K, Subbakrishna DK. Burden and coping in caregivers of persons with schizophrenia. Indian J of Psychiatry 2002;44(3):220-7.

25. Vasudeva S, Sekhar CK, Rao PG. Caregivers burden of patients with schizophrenia and bipolar disorder. A sectional study. Indian $\mathrm{J}$ of Psychological Medicine 2013;35(4):352-57.

26. Pinquart M, Sorensen S. Associations of stressors and uplifts of caregiving with caregiver burden and depressive mood: a meta-analysis. J Gerontol B Psychol Sci Soc Sci 2003;58(2):112-28.

27. Kessler RC, Price RH, Wortman CB. Social factors in psychopathology: stress, social support, and coping processes. Annual Review of Psychology 1985;36: 531-72.

28. Angermeyer NC. Normal deviance-changing norms under abnormal circumstances. In: Piehot P, Berner P, Wolf R, et al. eds. Psychiatry, the state of the art. vol 7. New York: Plenum. Epidemiology and Community Psychiatry 1985:473-9.

29. Noh S, Turner RJ. Living with psychiatric patients: implications for the mental health of family members. Social Science and Medicine 1987;25(3):263-72. 